\title{
INDECOMPOSABLE MODULES OF LARGE RANK OVER COHEN-MACAULAY LOCAL RINGS
}

\author{
WOLFGANG HASSLER, RYAN KARR, LEE KLINGLER, AND ROGER WIEGAND
}

\begin{abstract}
A commutative Noetherian local ring $(R, \mathfrak{m}, k)$ is called Dedekindlike provided $R$ is one-dimensional and reduced, the integral closure $\bar{R}$ is generated by at most 2 elements as an $R$-module, and $\mathfrak{m}$ is the Jacobson radical of $\bar{R}$. If $M$ is an indecomposable finitely generated module over a Dedekind-like ring $R$, and if $P$ is a minimal prime ideal of $R$, it follows from a classification theorem due to L. Klingler and L. Levy that $M_{P}$ must be free of rank 0, 1 or 2 .

Now suppose $(R, \mathfrak{m}, k)$ is a one-dimensional Cohen-Macaulay local ring that is not Dedekind-like, and let $P_{1}, \ldots, P_{t}$ be the minimal prime ideals of $R$. The main theorem in the paper asserts that, for each non-zero $t$-tuple $\left(n_{1}, \ldots, n_{t}\right)$ of non-negative integers, there is an infinite family of pairwise non-isomorphic indecomposable finitely generated $R$-modules $M$ satisfying $M_{P_{i}} \cong\left(R_{P_{i}}\right)^{\left(n_{i}\right)}$ for each $i$.
\end{abstract}

\section{$\S 1$. INTRODUCTION}

In 1911, E. Steinitz determined the structure of all finitely generated modules over Dedekind domains. This structure is so simple that one is tempted to try to generalize Steinitz's result to a larger class of commutative rings. Indeed, in a recent series of papers [KL1], [KL2], [KL3] L. Klingler and L. Levy presented a classification, up to isomorphism, of all finitely generated modules over a class of commutative rings they call "Dedekind-like".

We recall that a commutative Noetherian local $\operatorname{ring}(R, \mathfrak{m}, k)$ is Dedekind-like [KL1, Definition 2.5] provided $R$ is one-dimensional and reduced, the integral closure $\bar{R}$ is generated by at most 2 elements as an $R$-module, and $\mathfrak{m}$ is the Jacobson radical of $\bar{R}$. (In [KL2, (1.1.3)] a further requirement is imposed: If $\bar{R} / \mathfrak{m}$ is a field, then it is a separable extension of $k$. Klingler and Levy prove their classification theorem only under this additional hypothesis. In the present paper, however, we do not require that Dedekind-like rings satisfy this separability condition.) Although Dedekind-like rings are very close to their normalizations, their module structure is much more complicated than that of Dedekind domains. Klingler and Levy dash any hope of a further extension of their classification theorem by showing that, if $R$ is not a homomorphic image of a Dedekind-like ring or a special type

Received by the editors November 2, 2004 and, in revised form, October 14, 2005.

2000 Mathematics Subject Classification. Primary 13C05, 13E05, $13 \mathrm{H} 10$.

The first author's research was supported by a grant from the Fonds zur Förderung der wissenschaftichen Forschung, project number P16770-N12. The fourth author was partially supported by a grant from the National Science Foundation. The third author thanks the University of Nebraska-Lincoln, where much of the research was completed. 
of Artinian ring which they call a Klein ring, then $R$ must be "finite-length wild". This means, roughly speaking, that a classification of finite-length modules over $R$ would yield, for some field $k$, a classification of finite-dimensional modules over every finite-dimensional $k$-algebra. The apparent hopelessness of obtaining such a classification makes any further generalizations of Steinitz's result unlikely.

One of the peculiarities of Dedekind-like rings is that there is a bound on the torsion-free ranks of their indecomposable finitely generated modules; in fact, these torsion-free ranks are always bounded by two. Recently W. Hassler and R. Wiegand $[\mathrm{HaW}]$ constructed an indecomposable finitely generated module of torsion-free rank two over the cusp $k\left[\left[X^{2}, X^{3}\right]\right]$ (where $k$ is an arbitrary field) and over some related rings. The approach they used to build this module resembles the construction of rank-two indecomposable modules over unsplit Dedekind-like rings in [KL2]. Although the cusp $k\left[\left[X^{2}, X^{3}\right]\right]$ is not Dedekind-like, it is a Bass ring [LevW]; therefore all of its indecomposable finitely generated torsion-free modules have torsion-free rank one [B]. In this situation it is natural to ask whether there exist indecomposable finitely generated modules of even higher rank over $k\left[\left[X^{2}, X^{3}\right]\right]$ and over other non-Dedekind-like rings. The results of the current paper grew out of an attempt to answer this question.

In this paper all rings commute, and local and semi-local rings are assumed to be Noetherian. In order to state our main result, we need to define precisely what we mean by the "rank" of a module.

Definition 1.1. Let $R$ be a Noetherian ring with total quotient ring $K=$ nnonzerodivisors of $R\}^{-1} R$. A finitely generated $R$-module is called generically free if $K \otimes_{R} M$ is a projective $K$-module; equivalently, $M_{P}$ is $R_{P}$-free for every $P \in$ $\operatorname{Ass}(R)$. If $M_{P} \cong R_{P}^{\left(n_{P}\right)}$ for every $P \in \operatorname{Ass}(R)$, we say that the tuple $\left(n_{P}\right)_{P \in \operatorname{Ass}(R)}$ is the torsion-free rank of $M$. Further, we say that $M$ has constant rank $r$, if $M$ has torsion free rank $\left(n_{P}\right)_{P \in \operatorname{Ass}(R)}$, and $n_{P}=r$ for all $P \in \operatorname{Ass}(R)$.

If $R$ is Cohen-Macaulay, then $\operatorname{Ass}(R)$ consists only of the minimal primes $P_{1}, \ldots$, $P_{t}$ of $R$. Hence $K=K_{1} \times \ldots \times K_{t}$, where $K_{i}=R_{P_{i}}$. We are now prepared to state our main theorem:

Theorem 1.2 (Main Theorem). Let $(R, \mathfrak{m}, k)$ be a Cohen-Macaulay local ring of dimension one, and assume $R$ is not Dedekind-like. Let $K=K_{1} \times \ldots \times K_{t}$ be as above. For each non-zero t-tuple $\left(r_{1}, \ldots, r_{t}\right)$ of non-negative integers, there is an infinite family of pairwise non-isomorphic indecomposable finitely generated $R$ modules of torsion-free rank $\left(r_{1}, \ldots, r_{t}\right)$.

To prove this result we pass to an auxiliary ring $\Omega$ between $R$ and its normalization $\bar{R}$. We take cyclic modules over $\Omega$ as our building blocks, some of finite length and some of infinite length. These blocks undergo a process of "top-gluing" and "bottom-gluing", and eventually we obtain the desired indecomposable $R$-modules. An important tool for our construction is the theory of "separated covers" developed in [KL2]. By using separated covers, one can study finitely generated $R$-modules and their $R$-homomorphisms by means of $\Omega$-modules and $\Omega$-homomorphisms.

It is often possible to build large indecomposable torsion-free modules. Suppose $(R, \mathfrak{m}, k)$ is a one-dimensional reduced local ring with finite normalization $\bar{R}$. Then there is a bound on the ranks of all finitely generated torsion-free indecomposable $R$-modules if and only if $R$ has finite Cohen-Macaulay type, equivalently, $R$ satisfies 
the Drozd-Roĭter conditions [DR]:

(1) $\bar{R}$ is generated by at most 3 elements as an $R$-module, and

(2) $\frac{\mathfrak{m} \bar{R}+R}{R}$ is a cyclic $R$-module.

See $[C W W]$ for a survey article on this result. We note $[\mathrm{CWW}]$ that if a onedimensional reduced local ring $R$, with finite normalization, does not satisfy the Drozd-Roiter conditions, then there even exist finitely generated indecomposable torsion-free $R$-modules with arbitrarily large constant rank. Further results on the existence of large indecomposable torsion-free modules can be found in [LW1], [LW2].

The paper is organized as follows: $\S 2$ is devoted to the proof of the Main Theorem. In $\S 3$ we briefly discuss rings of arbitrary dimension and ponder the case of one-dimensional rings that are not Cohen-Macaulay.

\section{$\S 2$. Constructions in Dimension one}

We begin with the preparations for the proof of Theorem 1.2.

§2a. Module-finite overrings. Let $R$ be a commutative Noetherian ring (not necessarily one-dimensional or Cohen-Macaulay or local), with total quotient ring $K$. Given a faithful ideal $I$ of $R$, we note that $I$ must contain a non-zerodivisor of $R$, or else $I$ would be contained in the union of the associated primes of $R$ and hence in some associated prime of $R$. But this would imply that some non-zero element of $R$ annihilates $I$. Therefore $K I=K$, so the canonical isomorphism from $K \otimes_{R} I$ to $K I$ shows that each $R$-endomorphism of $I$ extends uniquely to a $K$ endomorphism of $K$. That is, we can make a canonical identification of $\operatorname{End}_{R}(I)$ with $\left(I:_{K} I\right)=\{\gamma \in K \mid \gamma I \subseteq I\}$. Now $I$ finitely generated as an $R$-module implies that $\operatorname{End}_{R}(I)=\left(I:_{K} I\right)$ is also finitely generated as an $R$-module, which makes $\left(I:_{K} I\right)$ integral over $R$ and hence contained in the normalization $\bar{R}$ of $R$. (Note that we are allowing for the possibility that $\bar{R}$ might not be finitely generated as an $R$-module.) We denote by $\mathrm{J}(R)$ the Jacobson radical of the ring $R$.

Lemma 2.1. Let $R$ be a one-dimensional, semi-local Cohen-Macaulay ring with total quotient ring $K$, and assume that $\bar{R} \neq R$. Then $R \subsetneq\left(\mathrm{J}(R):_{K} \mathrm{~J}(R)\right)$; that is, there is some element $\gamma \in \bar{R}-R$ such that $\gamma \mathrm{J}(R) \subseteq \mathrm{J}(R)$.

Proof. We can harmlessly assume that $R$ is indecomposable. Since the prime spectrum of an indecomposable ring must be connected, and a height zero maximal ideal would be both open and closed in $\operatorname{Spec}(R)$, it follows that every maximal ideal of $R$ must have height one. We claim that there is some maximal ideal $\mathfrak{m}$ such that $R_{\mathfrak{m}} \neq \overline{R_{\mathfrak{m}}}$. Suppose first that $R$ is reduced. Then $\overline{R_{\mathfrak{m}}}=\bar{R}_{\mathfrak{m}}$ for each maximal ideal $\mathfrak{m}$ by [HaW, Lemma 2.1]; in this case we choose any maximal ideal $\mathfrak{m}$ such that $R_{\mathfrak{m}} \neq \bar{R}_{\mathfrak{m}}$, proving the claim. If, on the other hand, $R$ is not reduced, we choose a non-zero element $x$ with $x^{2}=0$. Let $\mathfrak{m}$ be a maximal ideal such that (the image of) $x$ is non-zero in $R_{\mathfrak{m}}$. Since $R_{\mathfrak{m}}$ is Cohen-Macaulay of dimension one, there is a non-zerodivisor $t \in \mathfrak{m} R_{\mathfrak{m}}$, and then $\frac{x}{t^{n}} \in \overline{R_{\mathfrak{m}}}$ for every $n \geq 1$. Choosing $n$ so large that $x \notin R_{\mathfrak{m}} t^{n}$, we have $\frac{x}{t^{n}} \notin R_{\mathfrak{m}}$ as desired.

Thus we may assume that $(R, \mathfrak{m}, k)$ is local. We claim that $\mathfrak{m}$ does not have a direct summand isomorphic to $R$. Suppose, by way of contradiction, that $\mathfrak{m}=X \oplus Y$ with $X \cong R$. Then $X Y=0$, and since $X$ is faithful we have $Y=0$. But then 
$\mathfrak{m}=X \cong R$ is principal, which implies that $R$ is a discrete valuation ring, and hence $\bar{R}=R$, contradiction.

Since $\operatorname{depth}(R)=1$ we have $\operatorname{Hom}_{R}(k, R)=0$ and $\operatorname{Ext}_{R}^{1}(k, R) \neq 0$. Applying $\operatorname{Hom}_{R}\left(\_, R\right)$ to the short exact sequence $0 \rightarrow \mathfrak{m} \rightarrow R \rightarrow k \rightarrow 0$, we get an exact sequence

$$
0 \rightarrow R \rightarrow \operatorname{Hom}_{R}(\mathfrak{m}, R) \rightarrow \operatorname{Ext}_{R}^{1}(k, R) \rightarrow 0 .
$$

As shown above, $\mathfrak{m}$ does not have a direct summand isomorphic to $R$, so that the image of every $R$-homomorphism from $\mathfrak{m}$ to $R$ must be contained in the unique maximal ideal $\mathfrak{m}$ of $R$. That is, $\operatorname{Hom}_{R}(\mathfrak{m}, R)=\operatorname{End}_{R}(\mathfrak{m})=\left(\mathfrak{m}:_{K} \mathfrak{m}\right)$. Therefore, since $\operatorname{Ext}_{R}^{1}(k, R) \neq 0$, the exact sequence (2.1.1) shows that the inclusion $R \rightarrow$ $\left(\mathfrak{m}:_{K} \mathfrak{m}\right)$ is proper, as desired.

Proposition 2.2. Let $(R, \mathfrak{m}, k)$ be a one-dimensional Cohen-Macaulay local ring, and assume that $R$ is not Dedekind-like. Then at least one of the following holds.

(1) There is a local ring $(\Omega, \mathfrak{n}, k)$, with $R \subset \Omega \subseteq \bar{R}$, such that $\mathfrak{m}$ is the conductor of $R$ in $\Omega, \mathfrak{m} \subsetneq \mathfrak{n}$, and $\Omega$ is generated by 2 elements as an $R$-module. In particular, $\operatorname{dim}_{k}(\Omega / \mathfrak{m})=2$.

(2) $R$ is reduced, $\bar{R}$ is finitely generated as an $R$-module, $\mathfrak{m}$ is the conductor of $R$ in $\bar{R}, \mathfrak{m}=\mathrm{J}(\bar{R})$, and $\operatorname{dim}_{k}(\bar{R} / \mathfrak{m}) \geq 3$.

Proof. We first observe that, if $R$ is not reduced, then $\bar{R}$ is not finitely generated as an $R$-module. To see this, suppose $x$ is a non-zero nilpotent element. Choosing a non-zerodivisor $t \in \mathfrak{m}$, we get an infinite strictly ascending chain $R \frac{x}{t} \subset R \frac{x}{t^{2}} \subset$ $R \frac{x}{t^{3}} \subset \ldots$ of submodules of $\bar{R}$. (If $\frac{x}{t^{n+1}} \in R \frac{x}{t^{n}}$, write $\frac{x}{t^{n+1}}=r \frac{x}{t^{n}}$, getting $x(1-r t)=$ 0 , which forces $x=0$.)

In particular, if $R$ were equal to $\bar{R}$, then $R$ would be a discrete valuation domain, contradicting our assumption that $R$ is not Dedekind-like. Therefore $R \subsetneq \bar{R}$, and now Lemma 2.1 implies that $\Gamma:=\left(\mathfrak{m}:_{K} \mathfrak{m}\right)$ is a proper extension of $R$. Since $\mathfrak{m} \Gamma=\mathfrak{m}$, it follows that $\mathfrak{m}$ is the conductor of $R$ in $\Gamma$.

Suppose first that $\mathfrak{m} \neq \mathrm{J}(\Gamma)$. Since $R \cap \mathrm{J}(\Gamma)=\mathfrak{m}, \mathrm{J}(\Gamma) \nsubseteq R$, so we can choose an element $\delta \in \mathrm{J}(\Gamma)-R$. Since $\Gamma / \mathfrak{m}$ is Artinian, we can replace $\delta$ by one of its powers and assume that $\delta \notin R$ but $\delta^{2} \in R$. Then $\delta^{2} \in R \cap \mathrm{J}(\Gamma)=\mathfrak{m}$. We set $\Omega=R[\delta]=R+R \delta$. Then $\Omega$ is a ring and is two-generated as an $R$-module. Further, $\mathfrak{m}$ is the conductor of $R$ in $\Omega$. Clearly $\Omega$ is local, with maximal ideal $\mathfrak{n}:=\mathfrak{m}+R \delta \supsetneq \mathfrak{m}$. Therefore (1) holds.

Suppose instead that $\mathfrak{m}=\mathrm{J}(\Gamma)$. Since $\Gamma \cong \operatorname{End}_{R}(\mathfrak{m})$ (see the paragraph preceding Lemma 2.1), $\Gamma$ is finitely generated as an $R$-module. Thus $\Gamma$ is semilocal, and $\left(\mathrm{J}(\Gamma):_{K} \mathrm{~J}(\Gamma)\right)=\left(\mathfrak{m}:_{K} \mathfrak{m}\right)=\Gamma$. Since $\mathrm{J}(\Gamma)$ contains a non-zerodivisor, $\Gamma$ is CohenMacaulay, and now Lemma 2.1 implies that $\Gamma=\bar{\Gamma}=\bar{R}$. By the first paragraph of the proof, $R$ is reduced. If $\operatorname{dim}_{k}(\bar{R} / \mathfrak{m}) \leq 2$, then $R$ would be Dedekind-like (by Nakayama's lemma), contrary to our assumption. Therefore $\operatorname{dim}_{k}(\bar{R} / \mathfrak{m}) \geq 3$, and (2) holds.

For consistency with the notation in [KL1]-[KL3], we shall always write maps on the right for the remainder of $\S 2$. The proof of Theorem 1.2 divides naturally into the two cases of Proposition 2.2. Thus, we handle Case (1) in $\S 2 \mathrm{~b}$, and Case (2) in $\S 2 \mathrm{c}$. We retain the notation established in Definition 1.1 and Theorem 1.2. 


\section{$\S 2$ b. The ramified case (Case (1) of Proposition 2.2).}

Theorem 2.3. Let $(R, \mathfrak{m}, k)$ and $(\Omega, \mathfrak{n}, k)$ be as in Case (1) of Proposition 2.2, and let $r_{1}, \ldots, r_{t}$ be non-negative integers, not all zero. Then there is an infinite family of pairwise non-isomorphic finitely generated indecomposable $R$-modules of torsion-free rank $\left(r_{1}, \ldots, r_{t}\right)$.

Proof. Let $Q_{i}$ be the kernel of the composition $\Omega \hookrightarrow \bar{R} \hookrightarrow K \rightarrow K_{i}=R_{P_{i}} \rightarrow$ $R_{P_{i}} / P_{i} R_{P_{i}}$. (The $Q_{i}$ are the minimal prime ideals of $\Omega$.) Choose

$$
\delta \in \mathfrak{n}-\left(\mathfrak{m} \cup Q_{1} \cup \ldots \cup Q_{t}\right)
$$

Then $\delta$ is a unit of $K$ and therefore a non-zerodivisor of $\Omega$. Moreover, $\delta \notin R$ since $\mathfrak{n} \cap R=\mathfrak{m}$. Therefore $\bar{\delta}:=\delta+\mathfrak{m} \in \Omega / \mathfrak{m}-k$, so that $\Omega / \mathfrak{m}=k+k \bar{\delta}$. By Nakayama's lemma, $\Omega=R+R \delta$. Since $\bar{\delta}$ is nilpotent, we must have $\bar{\delta}^{2}=0$, that is, $\delta^{2} \in \mathfrak{m}$. We have a conductor square

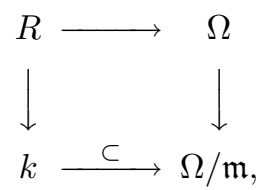

in which the right-hand vertical arrow is the natural surjection.

Fix an integer $n \geq \max \left\{r_{1}, \ldots, r_{t}\right\}$. We will construct an indecomposable finitely generated $R$-module $M_{n}$ with torsion-free rank $\left(r_{1}, \ldots, r_{t}\right)$ requiring exactly $4 n$ generators.

Since $K=K_{1} \times \ldots \times K_{t}$ is the total quotient ring of $R$ (cf. the paragraph preceding Theorem 1.2), we can choose an element $\lambda_{i} \in K_{i}^{\times} \cap R$ (where $K_{i}^{\times}$denotes the group of units of $K_{i}$ ) for each index $i \in\{1, \ldots, t\}$. For any subset $C \subseteq\{1, \ldots, t\}$, let $w_{C}:=\delta^{8} \cdot \sum_{i \notin C} \lambda_{i} \in \delta^{8} \Omega$; then the module $\Omega / w_{C} \Omega$ has torsion-free rank $\left(c_{1}, \ldots, c_{t}\right)$, where $c_{i}=1$ if $i \in C$ and $c_{i}=0$ otherwise. For each index $j, 1 \leq j \leq n$, let $C_{j}=\left\{i \mid 1 \leq i \leq t\right.$ and $\left.r_{i} \geq j\right\}$. If $1 \leq i \leq t$, then $i$ is an element of $C_{1} \cap \ldots \cap C_{r_{i}}$ but not an element of $C_{r_{i}+1} \cup \ldots \cup C_{n}$. Although the sets $C_{j}$ are not necessarily distinct, we see that for each $i \leq t$ there are exactly $r_{i}$ indices $j$ for which $i \in C_{j}$. It follows that the module $X_{1}:=\Omega / w_{C_{1}} \Omega \oplus \ldots \oplus \Omega / w_{C_{n}} \Omega$ has torsion-free rank $\left(r_{1}, \ldots, r_{t}\right)$. Moreover, since each $w_{C_{j}}$ is in $\mathfrak{m}$, the natural map $\Omega^{(n)} \rightarrow(\Omega / \mathfrak{m})^{(n)}$ induces a natural surjection $\nu_{1}: X_{1} \rightarrow(\Omega / \mathfrak{m})^{(n)}$, which induces a natural identification $X_{1} / \mathfrak{m} X_{1}=(\Omega / \mathfrak{m})^{(n)}$.

Next, let $X_{2}=\left(\Omega / \delta^{4} \mathfrak{m}\right)^{(n)}, X_{3}=\left(\Omega / \delta^{2} \mathfrak{m}\right)^{(n)}$, and $X_{4}=(\Omega / \mathfrak{m})^{(n)}$. Again, the natural map $\Omega^{(n)} \rightarrow(\Omega / \mathfrak{m})^{(n)}$ provides a natural surjection $\nu_{i}: X_{i} \rightarrow(\Omega / \mathfrak{m})^{(n)}$ and allows us to identify each $X_{i} / \mathfrak{m} X_{i}$ with $(\Omega / \mathfrak{m})^{(n)}=X_{4}$. Let $X=X_{1} \oplus X_{2} \oplus X_{3} \oplus X_{4}$ and $\nu=\nu_{1} \oplus \nu_{2} \oplus \nu_{3} \oplus \nu_{4}: X \rightarrow(\Omega / \mathfrak{m})^{(4 n)}$. We define an $R$-module $S$ by the following pullback square:

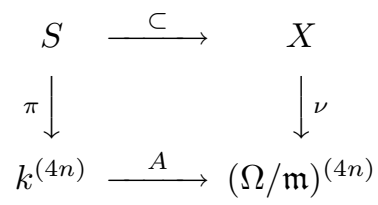


Here the elements of $k^{(4 n)}$ are viewed as row vectors, subjected to right multiplication by the matrix

$$
A:=\left(\begin{array}{cccc}
I & 0 & 0 & 0 \\
\bar{\delta} I & 0 & I & 0 \\
0 & I & 0 & 0 \\
0 & \bar{\delta} I & \bar{\delta} I & I
\end{array}\right),
$$

where $I$ denotes the $n \times n$ identity matrix.

In fact $S$ is a separated $R$-module [KL2, Definition 4.3], which just means that $S$ is an $R$-submodule of some $\Omega$-module (namely $X$ ). Also, $\Omega S=X$ (computed inside $X)$, as can be easily seen from the definition of $S$ as pullback in (2.3.2), together with the fact that the rows of $A$ span $(\Omega / \mathfrak{m})^{(4 n)}$ as an $\Omega / \mathfrak{m}$-module. The maps $\pi$ and $\nu$ in (2.3.2) have the same kernel, namely $\mathfrak{m} S=\mathfrak{m} \Omega S=\mathfrak{m} X$; therefore $\pi$ and $\nu$ are just the natural surjections $S \rightarrow S / \mathfrak{m} S$ and $X \rightarrow X / \mathfrak{m} X$. Moreover, there is a natural isomorphism $\Omega \otimes_{R} S \cong \Omega S=X$ by [KL2, Lemma 5.2]. (Actually, that lemma assumes that $R$ is a Dedekind-like ring, but for the proof of the direction we are applying here, the only facts used are that $R$ is local, its maximal ideal $\mathfrak{m}$ is the conductor, and $S / \mathfrak{m} S$ and $X / \mathfrak{m} X$ are free $k$ - and $\Omega / \mathfrak{m}$-modules, respectively, of the same rank.)

Next, multiplication by $\delta^{4}$ defines a homomorphism $\Omega \rightarrow \Omega / \delta^{4} \mathfrak{m}$ with kernel $\mathfrak{m}$ (since $\delta$ is a non-zerodivisor). This homomorphism, in turn, induces an injection $\xi: \Omega / \mathfrak{m} \rightarrow \Omega / \delta^{4} \mathfrak{m} ;$ moreover, $\operatorname{Im} \xi=\delta^{4} \Omega / \delta^{4} \mathfrak{m}$. We let $\sigma_{2}$ be the direct-sum map $\xi^{(n)}:(\Omega / \mathfrak{m})^{(n)} \rightarrow\left(\Omega / \delta^{4} \mathfrak{m}\right)^{(n)}=X_{2}$, so that $\sigma_{2}$ is injective, and $\operatorname{Im} \sigma_{2}=$ $\delta^{4} X_{2} \subseteq \mathfrak{m} X_{2}$. Similarly, multiplication by $\delta^{2}$ from $\Omega$ to $\Omega / \delta^{2} \mathfrak{m}$ induces an injection $\eta: \Omega / \mathfrak{m} \rightarrow \Omega / \delta^{2} \mathfrak{m}$ with $\operatorname{Im} \eta=\delta^{2} \Omega / \delta^{2} \mathfrak{m}$. We let $\sigma_{3}$ be the direct-sum map $\eta^{(n)}$ : $(\Omega / \mathfrak{m})^{(n)} \rightarrow\left(\Omega / \delta^{2} \mathfrak{m}\right)^{(n)}=X_{3}$, so that $\sigma_{3}$ is injective, and $\operatorname{Im} \sigma_{3}=\delta^{2} X_{3} \subseteq \mathfrak{m} X_{3}$. Since $\mathfrak{m} X_{2} \oplus \mathfrak{m} X_{3} \subset \mathfrak{m} X=\mathfrak{m} \Omega S=\mathfrak{m} S$, we obtain a commutative diagram:

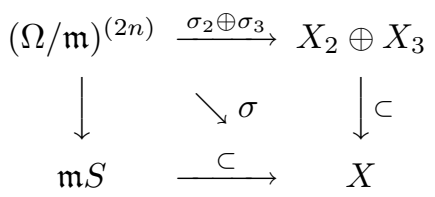

where $\sigma=\left(\begin{array}{cccc}0 & \sigma_{2} & 0 & 0 \\ 0 & 0 & \sigma_{3} & 0\end{array}\right)$.

Now let $B: k^{(2 n)} \rightarrow(\Omega / \mathfrak{m})^{(2 n)}$ be right multiplication by the matrix

$$
B=\left(\begin{array}{cc}
I & \bar{\delta} H \\
0 & I
\end{array}\right)
$$

where $I$ is the $n \times n$ identity matrix and $H$ is the indecomposable nilpotent $n \times n$ Jordan block:

$$
H:=\left(\begin{array}{ccccc}
0 & 1 & 0 & \ldots & 0 \\
0 & 0 & 1 & \ldots & 0 \\
\vdots & \vdots & \vdots & \ddots & \vdots \\
0 & 0 & 0 & \ldots & 1 \\
0 & 0 & 0 & \ldots & 0
\end{array}\right)
$$


Noting that $\operatorname{Im} \sigma \subset \mathfrak{m} X \subset S$, we define $\tau$ to be the composition $B \sigma: k^{(2 n)} \rightarrow S$, as in the following commutative diagram:

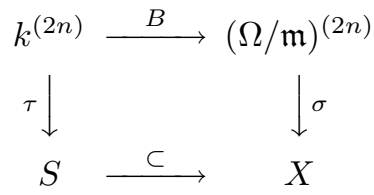

Finally, we define $M_{n}:=S / \operatorname{Im} \tau$.

Since $\operatorname{Im} \tau=\operatorname{Im}(B \sigma) \cong k^{(2 n)}$ is a torsion $R$-module, we see that $K \otimes_{R} M_{n} \cong$ $K \otimes_{R} S$. As noted above, $\Omega \otimes_{R} S \cong X$, so that

$$
K \otimes_{R} S \cong K \otimes_{\Omega} \Omega \otimes_{R} S \cong K \otimes_{\Omega} X .
$$

But $X_{2}, X_{3}$, and $X_{4}$ are torsion, being annihilated by $\delta^{6}$, so that

$K \otimes_{\Omega} X \cong K \otimes_{\Omega} X_{1}=K \otimes_{\Omega}\left(\Omega / w_{C_{1}} \Omega \oplus \ldots \oplus \Omega / w_{C_{n}} \Omega\right) \cong K / w_{C_{1}} K \oplus \ldots \oplus K / w_{C_{n}} K$.

By the choice of the elements $w_{C_{j}}, M_{n}$ has torsion-free rank $\left(r_{1}, \ldots, r_{t}\right)$.

Since $\operatorname{Im} \tau \subseteq \mathfrak{m} S$, it follows that $M_{n} / \mathfrak{m} M_{n}=(S / \operatorname{Im} \tau) /(\mathfrak{m} S / \operatorname{Im} \tau) \cong S / \mathfrak{m} S=$ $S / \mathfrak{m} X \cong k^{(4 n)}$, by (2.3.2). Thus the sequence $\left\{M_{n} \mid n \geq \max \left\{r_{1}, \ldots, r_{t}\right\}\right\}$ is an infinite family of pairwise non-isomorphic $R$-modules, each with torsion-free rank $\left(r_{1}, \ldots, r_{t}\right)$.

To complete the proof of Theorem 2.3, we show that $M_{n}$ is indecomposable. Suppose that $f$ is an idempotent $R$-endomorphism of $M_{n}$ and that $f$ is not surjective. We will show that $f=0$. By Nakayama's lemma, it will suffice to show that $\operatorname{Im} f \subseteq \mathfrak{m} M_{n}$.

One easily checks that the $R$-submodule $\operatorname{Im} B$ of $(\Omega / \mathfrak{m})^{(2 n)}$ does not contain a non-zero $\Omega$-submodule of $(\Omega / \mathfrak{m})^{(2 n)}$. Hence no non-zero $\Omega$-submodule of $X$ is contained in $\operatorname{Im} \tau$. Since, in addition, $\operatorname{Im} \tau \subseteq \mathfrak{m} S$, the module $S$ is a separated cover of $M_{n}$ [KL2, Lemma 4.9]. Therefore, by [KL2, Theorem 4.12], $f$ lifts to an $R$-endomorphism $\theta$ of $S$, and $\theta$ in turn extends to an $\Omega$-endomorphism $\theta^{\prime}=1_{\Omega} \otimes_{R} \theta$ of $\Omega \otimes_{R} S=X$. Then $\theta$ and $\theta^{\prime}$ induce endomorphisms $\bar{\theta}$ and $\bar{\theta}^{\prime}$ of $S / \mathfrak{m} S=k^{(4 n)}$ and $X / \mathfrak{m} X=(\Omega / \mathfrak{m})^{(4 n)}$, respectively. We assemble these maps in the following cube, in which the inner and outer squares are the pullback diagram (2.3.2):

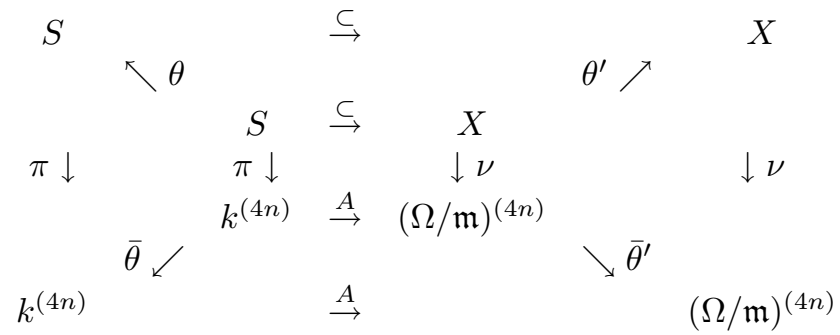

Since all faces except possibly the bottom trapezoid commute, and since the map $\pi: S \rightarrow k^{(4 n)}$ is surjective, it follows that the bottom trapezoid commutes as well, that is, $\bar{\theta} A=A \bar{\theta}^{\prime}$.

The map $\bar{\theta}: k^{(4 n)} \rightarrow k^{(4 n)}$ is right multiplication by a $4 n \times 4 n$ matrix over $k$, which we write in block form as $\left(P_{i j}\right)$, where $1 \leq i, j \leq 4$ and each $P_{i j}$ is an $n \times n$ matrix over $k$. Similarly, we can represent the map $\theta^{\prime}$ as a matrix in block form $\left(Q_{i j}\right)$, where again $1 \leq i, j \leq 4$ and each $Q_{i j}$ is a map from $X_{i}$ to $X_{j}$. Now $Q_{i j}$ induces a map $\bar{Q}_{i j}: X_{i} / \mathfrak{m} X_{i} \rightarrow X_{j} / \mathfrak{m} X_{j}$. Since $X_{i} / \mathfrak{m} X_{i}=(\Omega / \mathfrak{m})^{(n)}$, each 
$\bar{Q}_{i j}$ can be viewed as an $n \times n$ matrix over $\Omega / \mathfrak{m}$, and the map $\bar{\theta}^{\prime}$ becomes right multiplication by the $4 n \times 4 n$ block matrix $\left(\bar{Q}_{i j}\right)$. In fact, the homomorphism $\nu$ is a diagonal map - reduction modulo $\mathfrak{m}$. Therefore, viewing the map $\theta^{\prime}$ as a matrix of maps between cyclic indecomposable $\Omega$-modules (via the given directsum decomposition of $X$ ), we see that the matrix $\left(\bar{Q}_{i j}\right)$ can be obtained from the matrix $\left(Q_{i j}\right)$ by simply reducing all entries modulo $\mathfrak{m}$.

We can say more about some of the matrices $\bar{Q}_{i j}$. Since $\delta^{6} X_{2}=0$ and $Q_{21}$ : $X_{2} \rightarrow X_{1}$, we see that $\delta^{6} \cdot \operatorname{Im} Q_{21}=0$. But $X_{1}=\Omega / w_{C_{1}} \Omega \oplus \ldots \oplus \Omega / w_{C_{n}} \Omega$, and $w_{C_{i}} \Omega \subseteq \delta^{8} \Omega$ for each index $i$. Since $\delta$ is a regular element of $\Omega$, we see that $\operatorname{Im} Q_{21} \subseteq \delta^{2} X_{1} \subseteq \mathfrak{m} X_{1}$. That is, the entries of $Q_{21}$ reduced modulo $\mathfrak{m}$ are 0 , and hence $\bar{Q}_{21}=0$. Similar arguments show that in fact $\bar{Q}_{i j}=0$ whenever $i>j$. In other words, the matrix $\left(\bar{Q}_{i j}\right)$ is block upper triangular. The equation $\bar{\theta} A=A \bar{\theta}^{\prime}$ now reads as follows:

$$
\begin{aligned}
& \left(\begin{array}{cccc}
P_{11}+\bar{\delta} P_{12} & P_{13}+\bar{\delta} P_{14} & P_{12}+\bar{\delta} P_{14} & P_{14} \\
P_{21}+\bar{\delta} P_{22} & P_{23}+\bar{\delta} P_{24} & P_{22}+\bar{\delta} P_{24} & P_{24} \\
P_{31}+\bar{\delta} P_{32} & P_{33}+\bar{\delta} P_{34} & P_{32}+\bar{\delta} P_{34} & P_{34} \\
P_{41}+\bar{\delta} P_{42} & P_{43}+\bar{\delta} P_{44} & P_{42}+\bar{\delta} P_{44} & P_{44}
\end{array}\right) \\
= & \left(\begin{array}{cccc}
\bar{Q}_{11} & \bar{Q}_{12} & \bar{Q}_{13} & \bar{Q}_{14} \\
\bar{\delta} \bar{Q}_{11} & \bar{\delta} \bar{Q}_{12} & \bar{\delta} \bar{Q}_{13}+\bar{Q}_{33} & \bar{\delta} \bar{Q}_{14}+\bar{Q}_{34} \\
0 & \bar{Q}_{22} & \bar{Q}_{23} & \bar{Q}_{24} \\
0 & \bar{\delta} \bar{Q}_{22} & \bar{\delta} \bar{Q}_{23}+\bar{\delta} \bar{Q}_{33} & \bar{\delta} \bar{Q}_{24}+\bar{\delta} \bar{Q}_{34}+\bar{Q}_{44}
\end{array}\right) .
\end{aligned}
$$

Working column by column in order, comparing entries and using the fact that $\{1, \bar{\delta}\}$ is linearly independent over $k$, one shows that the $P_{i j}$ have entries in $k$, and that $\bar{\delta}^{2}=0$. We see easily that $P_{11}=P_{22}=P_{33}=P_{44}$, and for convenience we put $\Delta:=P_{11}$. Moreover, $\bar{Q}_{i i}=\Delta+\bar{\delta} V_{i}$ for suitable matrices $V_{i}$ with entries in $k$. Thus, if we reduce $\theta^{\prime}$ modulo $\mathfrak{n}=\mathfrak{m}+\delta \Omega$, the resulting endomorphism of $X / \mathfrak{n} X=k^{(4 n)}$ is right multiplication by a block upper-triangular matrix over $k$ with four identical $n \times n$ diagonal blocks $\Delta$.

We shall show that in fact $\Delta$ itself is upper triangular with constant diagonal. To see this, we look at the matrix $B$ and its relation to $\theta$ and $\theta^{\prime}$. Since the map $\theta: S \rightarrow S$ induces the $R$-endomorphism $f$ of $M_{n}=S / \operatorname{Im} \tau$, it follows that $(\operatorname{Im} \tau) \theta \subseteq \operatorname{Im} \tau$. Therefore $\theta$ can be lifted to an $R$-homomorphism $\tilde{\theta}: k^{(2 n)} \rightarrow k^{(2 n)}$ such that $\tau \theta=\tilde{\theta} \tau$. Moreover, since $B$ is invertible, $\operatorname{Im}(B \sigma)$ generates $\operatorname{Im} \sigma$ as an $\Omega$ submodule of $X$. Since the map $\theta^{\prime}: X \rightarrow X$ extends $\theta$, it follows that $(\operatorname{Im}(\sigma)) \theta^{\prime} \subseteq$ $\operatorname{Im}(\sigma)$. Therefore $\theta^{\prime}$ lifts to an $\Omega$-homomorphism $\tilde{\theta}^{\prime}:(\Omega / \mathfrak{m})^{(2 n)} \rightarrow(\Omega / \mathfrak{m})^{(2 n)}$ such that $\sigma \theta^{\prime}=\tilde{\theta}^{\prime} \sigma$. (A preliminary peek at the diagram below is helpful here.) These maps yield a cube

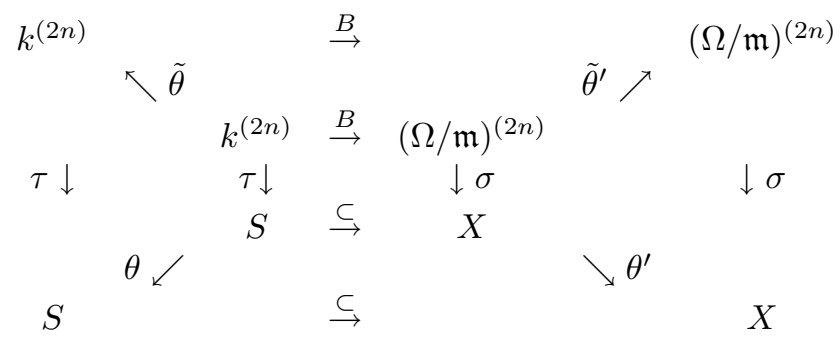


in which the left, right and bottom trapezoids commute. Also, the inside and outside squares commute by (2.3.5). Since the map $\sigma$ is injective, it follows that the top trapezoid commutes as well, and we have the identity $\tilde{\theta} B=B \tilde{\theta}^{\prime}$.

The map $\tilde{\theta}$ is right multiplication by a $2 n \times 2 n$ matrix over $k$. We write this matrix in block form as $\left(R_{i j}\right)$, where $1 \leq i, j \leq 2$, and each $R_{i j}$ is an $n \times n$ matrix over $k$. Similarly, $\tilde{\theta}^{\prime}$ is right multiplication by a matrix $\left(\begin{array}{cc}\tilde{Q}_{22} & \tilde{Q}_{23} \\ \tilde{Q}_{32} & \tilde{Q}_{33}\end{array}\right)$, where each $\tilde{Q}_{i j}$ is an $n \times n$ matrix over $(\Omega / \mathfrak{m})$. (The strange numbering is for compatibility with $\sigma$, whose image is contained in $X_{2} \oplus X_{3}$.) We have already written $\theta^{\prime}$ as a matrix $\left(Q_{i j}\right)$, where each $Q_{i j}$ is a map from $X_{i}$ to $X_{j}$.

As above, we can say more about some of the matrices $\tilde{Q}_{i j}$. Since the homomorphism $\sigma$ is the diagonal map $\sigma_{2} \oplus \sigma_{3}$, commutativity of the right-hand trapezoid of (2.3.7) implies that $\tilde{Q}_{23} \sigma_{3}=\sigma_{2} Q_{23}$. Now $\operatorname{Im} \sigma_{2}=\delta^{4} X_{2}$, so that $\operatorname{Im}\left(\sigma_{2} Q_{23}\right) \subseteq \delta^{4}\left(X_{2}\right) Q_{23} \subseteq \delta^{4} X_{3}=0$ (since $\delta^{2} \in \mathfrak{m}$ and $\left.X_{3}=\left(\Omega / \delta^{2} \mathfrak{m}\right)^{(n)}\right)$. But $\sigma_{3}$ is injective, so it follows that $\tilde{Q}_{23}=0$; that is, the matrix $\left(\tilde{Q}_{i j}\right)$ is block lower triangular.

Similarly, for the diagonal block $Q_{22}$, commutativity of the right-hand trapezoid of (2.3.7) yields the equation $\tilde{Q}_{22} \sigma_{2}=\sigma_{2} Q_{22}$, but now we want to use the fact that $\sigma_{2}$ is itself a diagonal map $\xi^{(n)}:(\Omega / \mathfrak{m})^{(n)} \rightarrow\left(\Omega / \delta^{4} \mathfrak{m}\right)^{(n)}$. Thus, if we write $Q_{22}=\left(q_{i j}\right)$ and $\tilde{Q}_{22}=\left(\tilde{q}_{i j}\right)$, then commutativity of $(2.3 .7)$ yields equal maps $\tilde{q}_{i j} \xi=\xi q_{i j}$ from $\Omega / \mathfrak{m}$ to $\Omega / \delta^{4} \mathfrak{m}$, for each pair of indices $i, j(1 \leq i, j \leq n)$. Now we can view $\tilde{q}_{i j}$ as multiplication by an element $\tilde{q}_{i j} \in \Omega$ (modulo the ideal $\mathfrak{m}$ ), and $q_{i j}$ as multiplication by an element $q_{i j} \in \Omega$ (modulo the ideal $\delta^{4} \mathfrak{m}$ ). Then the equality of maps $\tilde{q}_{i j} \xi=\xi q_{i j}$ implies that $\tilde{q}_{i j}-q_{i j}$ annihilates the module $\operatorname{Im} \xi=\delta^{4} \Omega / \delta^{4} \mathfrak{m} \cong \Omega / \mathfrak{m}$; that is, $\tilde{q}_{i j}-q_{i j} \in \mathfrak{m}$. In other words, reducing the entries of the matrix $Q_{22}$ modulo $\mathfrak{m}$ yields exactly the matrix $\tilde{Q}_{22}$. But we have already noted that $Q_{22}$ modulo $\mathfrak{m}$ equals the matrix $\bar{Q}_{22}$. Therefore $\tilde{Q}_{22}=\bar{Q}_{22}=\Delta+\bar{\delta} V_{2}$. By a similar argument, $\tilde{Q}_{33}=\bar{Q}_{33}=\Delta+\bar{\delta} V_{3}$.

Since $\bar{\delta}^{2}=0$, the equation $\tilde{\theta} B=B \tilde{\theta}^{\prime}$, written in matrix form, now reads

$$
\left(\begin{array}{ll}
R_{11} & \bar{\delta} R_{11} H+R_{12} \\
R_{21} & \bar{\delta} R_{21} H+R_{22}
\end{array}\right)=\left(\begin{array}{cc}
\Delta+\bar{\delta}\left(V_{2}+H \tilde{Q}_{32}\right) & \bar{\delta} H \Delta \\
\tilde{Q}_{32} & \Delta+\bar{\delta} V_{3}
\end{array}\right) .
$$

Since the matrices $R_{i j}$ have entries in $k$, the $(1,1)$-entries show that $R_{11}=\Delta$, while the (1,2)-entries show that $R_{11} H=H \Delta$. Thus $\Delta H=H \Delta$; since $H$ is nonderogatory, $\Delta \in k[H]$. In particular, $\Delta$ is an upper-triangular matrix over $k$, with constant diagonal. Therefore, when we reduce $\theta^{\prime}$ modulo $\mathfrak{n}=\mathfrak{m}+\delta \Omega$, the resulting endomorphism of $X / \mathfrak{n} X=k^{(4 n)}$ is right multiplication by an upper-triangular matrix $\Xi$ with constant diagonal.

On the other hand, $\theta^{\prime}$ restricts to the $R$-endomorphsim $\theta$ of $S$, which in turn induces the $R$-endomorphism $f$ of $M_{n}$. But $f$ is not surjective, by assumption, and hence $\theta$ cannot be surjective. If $\theta^{\prime}$ were surjective, it would induce a surjective endomorphism of the Noetherian module $X / S$ and hence an automorphism of $X / S$, but since $(S) \theta^{\prime}=\operatorname{Im} \theta \subseteq S$, this would force $\operatorname{Im} \theta=S$, a contradiction. Therefore $\theta^{\prime}$ cannot be surjective either. Then, by Nakayama's lemma, the endomorphism of $X / \mathfrak{n} X=k^{(4 n)}$ induced by $\theta^{\prime}$ is not surjective. Since this endomorphism is given by right multiplication by the upper-triangular matrix $\Xi$ above, we see that the constant element on the diagonal must be 0 , and hence $\Xi$ is a nilpotent $4 n \times 4 n$ matrix. That is, $\operatorname{Im}\left(\theta^{\prime}\right)^{4 n} \subseteq \mathfrak{n} X=(\mathfrak{m}+R \delta) X$. Since $\delta^{2} \in \mathfrak{m}$, we have $\operatorname{Im} \theta^{8 n} \subseteq$ 
$\operatorname{Im}\left(\theta^{\prime}\right)^{8 n} \subseteq \mathfrak{m} X=\mathfrak{m} S$. Now $\theta^{8 n}$ induces the map $f^{8 n}=f$ on $M_{n}$. Therefore $\operatorname{Im} f \subseteq \mathfrak{m} M_{n}$, as desired.

§2c. The unramified case (Case (2) of Proposition 2.2). Let the notation be as in Theorem 1.2. In particular $K=K_{1} \times \ldots \times K_{t}$ is the total quotient ring and $\bar{R}$ the normalization of $R$. Our goal is the following theorem, which will complete the proof of Theorem 1.2.

Theorem 2.4. Let $(R, \mathfrak{m}, k)$ and $\bar{R}$ be as in Case (2) of Proposition 2.2. Then, for each t-tuple $\left(r_{1}, \ldots, r_{t}\right)$ of non-negative integers, there is an infinite family of pairwise non-isomorphic finitely generated indecomposable $R$-modules of torsionfree rank $\left(r_{1}, \ldots, r_{t}\right)$.

Proof. Put $D:=\bar{R} / \mathfrak{m}$. Since $\mathfrak{m}=\mathrm{J}(\bar{R})$, we have $D=F_{1} \times \ldots \times F_{m}$, where each $F_{i}$ is a field of finite degree over $k$. We number the components so that $\left[F_{1}: k\right] \geq \ldots \geq\left[F_{m}: k\right]$. Since $R$ is reduced and $\bar{R}$ is finitely generated as an $R$-module, $\bar{R}$ is a finite product of semilocal Dedekind domains, so that $m$ (the number of maximal ideals of $\bar{R}$ ) is greater than or equal to $t$ (the number of minimal prime ideals of $\bar{R}$ ). Most possibilities for $R$ are covered by a single construction ("the basic case"), which we present in detail. The remaining cases seem to require different constructions, which we outline at the end of this proof.

The basic case. For the basic construction we shall assume that, at one extreme, if $m=1$ (so that $\left[F_{1}: k\right] \geq 3$ ) and the characteristic of $F_{1}$ is 2 , then $F_{1}$ is a separable extension of $k$. At the other extreme we shall assume that, if $\left[F_{1}: k\right]=1$ (so that $m \geq 3$ ), then $|k|>3$. With these additional assumptions, we claim that there is a unit $\bar{u} \in D$ such that the set $\left\{1, \bar{u}, \bar{u}^{2}\right\}$ is linearly independent over $k$. If $m=1$, then the assumed restriction on $F_{1}$ ensures that there is an element $\bar{u} \in F_{1}$ of degree at least 3 over $k$. If $\left[F_{1}: k\right]=1$, then the assumed restriction on $k$ ensures that we can take $\bar{u}=(a, b, c, 1, \ldots, 1)$ for some distinct non-zero elements $a, b, c \in k$. (By a Vandermonde determinant argument it follows that $1, \bar{u}, \bar{u}^{2}$ are linearly independent over $k$.) This leaves the case where $m>1$ and $\left[F_{1}: k\right]>1$. In this case we put $\bar{u}=(\alpha, 1, \ldots, 1)$, where $\alpha$ is any element of $F_{1}-k$. If $p, q, r \in k$ with $p \bar{u}^{2}+q \bar{u}+r=0$, then both $\alpha$ and 1 are roots of the polynomial $p X^{2}+q X+r$, and it follows easily that $p=q=r=0$. This proves the claim.

Choose an element $u \in \bar{R}$ whose image in $D$ is $\bar{u}$, and set $\Omega:=R[u]$. Clearly $\mathfrak{m}$ is an ideal of $\Omega$ and hence is the conductor of $R$ in $\Omega$. Now $\Omega / \mathfrak{m}$ is a $k$-subalgebra of $D$ and therefore is a finite-dimensional semisimple $k$-algebra. Since $\mathfrak{m} \subseteq \mathrm{J}(\Omega)$, it follows that $\mathfrak{m}=\mathrm{J}(\Omega)$. Thus we again obtain the conductor square (2.3.1) for $R$.

As in the proof of Theorem 2.3, choose an element $\lambda_{i} \in K_{i}^{\times} \cap R$ for each in$\operatorname{dex} i$. For any subset $C \subseteq\{1, \ldots, t\}$ let $w_{C}:=\sum_{i \notin C} \lambda_{i}$. Also, choose subsets $C_{1}, \ldots, C_{n} \subseteq\{1, \ldots, t\}$ such that $i$ is in exactly $r_{i}$ sets $C_{j}$ for each $i$. The module $X_{1}:=\Omega / w_{C_{1}} \mathfrak{m}^{5} \oplus \ldots \oplus \Omega / w_{C_{n}} \mathfrak{m}^{5}$ then has torsion-free rank $\left(r_{1}, \ldots, r_{t}\right)$. The natural map $\Omega^{(n)} \rightarrow(\Omega / \mathfrak{m})^{(n)}$ allows us to make the identification $X_{1} / \mathfrak{m} X_{1}=(\Omega / \mathfrak{m})^{(n)}$.

The inclusion $\Omega / \mathfrak{m} \subseteq \bar{R} / \mathfrak{m}=D$ (of modules over the semisimple $k$-algebra $\Omega / \mathfrak{m}$ ) splits, so there is an $\Omega$-submodule $Y \subseteq D$, such that $D / Y \cong \Omega / \mathfrak{m}$. Since $\mathfrak{m}$ is a principal ideal of $\bar{R}$ generated by a non-zerodivisor, we have $\mathfrak{m}^{s} / \mathfrak{m}^{s+1} \cong \bar{R} / \mathfrak{m}=D$, for each positive integer $s$. Thus, for each positive integer $s$, there exists an $\Omega$ module $Y_{s}$ such that

$$
\mathfrak{m}^{s} \subseteq Y_{s} \subseteq \mathfrak{m}^{s-1} \text { and } \mathfrak{m}^{s-1} / Y_{s} \cong \Omega / \mathfrak{m}
$$


Put $X_{2}:=\left(\Omega / Y_{4}\right)^{(n)}$ and $X_{3}:=\left(\Omega / Y_{2}\right)^{(n)}$. The natural map $\Omega^{(n)} \rightarrow(\Omega / \mathfrak{m})^{(n)}$ allows the identifications $X_{2} / \mathfrak{m} X_{2}=(\Omega / \mathfrak{m})^{(n)}$ and $X_{3} / \mathfrak{m} X_{3}=(\Omega / \mathfrak{m})^{(n)}$. Let $X=X_{1} \oplus X_{2} \oplus X_{3}$, and let $\nu: X \rightarrow X / \mathfrak{m} X=(\Omega / \mathfrak{m})^{(3 n)}$ be the natural map.

We define an $R$-module $S$ by the following pullback square:

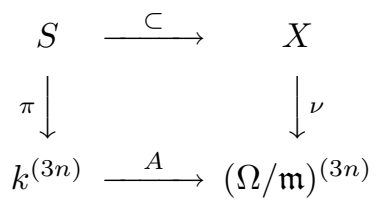

Here the elements of $k^{(3 n)}$ are viewed as row vectors, subjected to right multiplication by the matrix

$$
A:=\left(\begin{array}{ccc}
I & 0 & 0 \\
\bar{u} I & I & 0 \\
0 & \bar{u} I & I
\end{array}\right),
$$

where $I$ denotes the $n \times n$ identity matrix. As in the proof of Theorem 2.3,S is a separated $R$-module, and there is a natural isomorphism $\Omega \otimes_{R} S \cong \Omega S=X$ by [KL2, Lemma 5.2].

Since $\mathfrak{m}^{3} / Y_{4} \cong \Omega / \mathfrak{m} \cong \mathfrak{m} / Y_{2}$, we can fix injections $\xi: \Omega / \mathfrak{m} \rightarrow \Omega / Y_{4}$ and $\eta:$ $\Omega / \mathfrak{m} \rightarrow \Omega / Y_{2}$ such that $\operatorname{Im} \xi=\mathfrak{m}^{3} / Y_{4}$ and $\operatorname{Im} \eta=\mathfrak{m} / Y_{2}$. Then let $\sigma_{2}$ be the directsum map $\xi^{(n)}:(\Omega / \mathfrak{m})^{(n)} \rightarrow\left(\Omega / Y_{4}\right)^{(n)}=X_{2}$, so that $\sigma_{2}$ is injective, and $\operatorname{Im} \sigma_{2}=$ $\left(\mathfrak{m}^{3} / Y_{4}\right)^{(n)}=\mathfrak{m}^{3} X_{2}$. Similarly, let $\sigma_{3}$ be the direct-sum map $\eta^{(n)}:(\Omega / \mathfrak{m})^{(n)} \rightarrow$ $\left(\Omega / Y_{2}\right)^{(n)}=X_{3}$, so that $\sigma_{3}$ is injective, and $\operatorname{Im} \sigma_{3}=\left(\mathfrak{m} / Y_{2}\right)^{(n)}=\mathfrak{m} X_{3}$. Thus, $\operatorname{Im} \sigma_{2} \oplus \operatorname{Im} \sigma_{3}=\mathfrak{m}^{3} X_{2} \oplus \mathfrak{m} X_{3} \subset \mathfrak{m} X=\mathfrak{m} \Omega S=\mathfrak{m} S$, so we again get the commutative diagram (2.3.3), where now $\sigma=\left(\begin{array}{ccc}0 & \sigma_{2} & 0 \\ 0 & 0 & \sigma_{3}\end{array}\right)$.

Let $B: k^{(2 n)} \rightarrow \Omega^{(2 n)}$ be right multiplication by the matrix

$$
B=\left(\begin{array}{cc}
H & I \\
\bar{u}^{2} I & \bar{u} I
\end{array}\right)
$$

where $I$ is the $n \times n$ identity matrix, and $H$ is the nilpotent matrix in (2.3.4). Again, we define $\tau: k^{(2 n)} \rightarrow S$ by diagram (2.3.5) and put $M_{n}:=S / \operatorname{Im} \tau$. As in the proof of Theorem $2.3, K \otimes_{R} M_{n} \cong K / w_{C_{1}} K \oplus \ldots \oplus K / w_{C_{n}} K$, and hence $M_{n}$ has torsion-free rank $\left(r_{1}, \ldots, r_{t}\right)$.

Also as in the proof of Theorem 2.3, since $\operatorname{Im} \tau \subseteq \mathfrak{m} S$, it follows that $M_{n} / \mathfrak{m} M_{n}=$ $(S / \operatorname{Im} \tau) /(\mathfrak{m} S / \operatorname{Im} \tau) \cong S / \mathfrak{m} S=S / \mathfrak{m} X \cong k^{(3 n)}$, by (2.4.2). Thus, the sequence of $R$-modules $M_{n}$ (for $n \geq \max \left\{r_{1}, \ldots, r_{t}\right\}$ ) is an infinite family of pairwise nonisomorphic $R$-modules, each with torsion-free rank $\left(r_{1}, \ldots, r_{t}\right)$.

The proof of the basic case will be complete once we verify the indecomposability of the $R$-module $M_{n}$ just constructed. The proof that $M_{n}$ is indecomposable is much the same as in Theorem 2.3; we summarize the argument and focus only on the points where slight changes are needed. Thus, we suppose that $f$ is an idempotent $R$-endomorphism of $M_{n}$ and that $f$ is not surjective, and we show that $\operatorname{Im} f \subseteq \mathfrak{m} M_{n}$.

As in the proof of Theorem 2.3, one easily checks, from the form of the matrix $B$, that no non-zero $\Omega$-submodule of $X$ is contained in $\operatorname{Im} \tau$. Since $\operatorname{Im} \tau \subseteq \mathfrak{m} S$, it follows that $S$ is a separated cover of $M_{n}$. Thus $f$ lifts to an $R$-endomorphism $\theta$ of $S$, which in turn lifts to an $\Omega$-endomorphism $\theta^{\prime}$ of $X$. The maps $\theta$ and $\theta^{\prime}$ then induce endomorphisms $\bar{\theta}$ and $\bar{\theta}^{\prime}$ of $S / \mathfrak{m} S=k^{(3 n)}$ and $X / \mathfrak{m} X=(\Omega / \mathfrak{m})^{(3 n)}$, respectively, yielding a commutative cube similar to (2.3.6), the only difference 
being that " $(4 n)$ " is replaced by " $(3 n)$ " everywhere. The bottom trapezoid of this diagram gives the identity $\bar{\theta} A=A \bar{\theta}^{\prime}$.

We write $\bar{\theta}$ as right multiplication by the block matrix $\left(P_{i j}\right)$, where $1 \leq i, j \leq 3$, and each $P_{i j}$ is an $n \times n$ matrix over $k$. Similarly, we write $\theta^{\prime}$ as a matrix in block form $\left(Q_{i j}\right)$, where $1 \leq i, j \leq 3$, and each $Q_{i j}$ is a map from $X_{i}$ to $X_{j}$. Reduced modulo $\mathfrak{m}$, each $Q_{i j}$ becomes an $n \times n$ matrix $\bar{Q}_{i j}$ over $\Omega / \mathfrak{m}$, right multiplication by which is the induced map from $X_{i} / \mathfrak{m} X_{i} \cong(\Omega / \mathfrak{m})^{(n)}$ to $X_{j} / \mathfrak{m} X_{j} \cong(\Omega / \mathfrak{m})^{(n)}$.

As before, we will show that the matrix $\left(\bar{Q}_{i j}\right)$ is block upper triangular. Since $\mathfrak{m}^{2} \subseteq Y_{2}$ by (2.4.1), $\mathfrak{m}^{2} X_{3}=0$; therefore $\mathfrak{m}^{2}$ annihilates $\operatorname{Im} Q_{32} \subseteq X_{2}$. But $X_{2}=$ $\left(\Omega / Y_{4}\right)^{(n)}$, and $Y_{4} \subseteq \mathfrak{m}^{3}$ by (2.4.1). It follows that $\operatorname{Im} Q_{32} \subseteq\left(\mathfrak{m} / Y_{4}\right)^{(n)}=\mathfrak{m} X_{2}$. Thus $\bar{Q}_{32}=0$. Similarly, since $\mathfrak{m}^{4} X_{2}=\mathfrak{m}^{4} X_{3}=0$ while $X_{1}=\Omega / w_{C_{1}} \mathfrak{m}^{5} \oplus \ldots \oplus$ $\Omega / w_{C_{n}} \mathfrak{m}^{5}$, it follows easily that $\operatorname{Im} Q_{21}$ and $\operatorname{Im} Q_{31}$ are contained in $\mathfrak{m} X_{1}$; therefore $\bar{Q}_{21}=\bar{Q}_{31}=0$, as desired. The equation $\bar{\theta} A=A \bar{\theta}^{\prime}$ now looks like this:

$$
\left(\begin{array}{ccc}
P_{11}+\bar{u} P_{12} & P_{12}+\bar{u} P_{13} & P_{13} \\
P_{21}+\bar{u} P_{22} & P_{22}+\bar{u} P_{23} & P_{23} \\
P_{31}+\bar{u} P_{32} & P_{32}+\bar{u} P_{33} & P_{33}
\end{array}\right)=\left(\begin{array}{ccc}
\bar{Q}_{11} & \bar{Q}_{12} & \bar{Q}_{13} \\
\bar{u} \bar{Q}_{11} & \bar{u} \bar{Q}_{12}+\bar{Q}_{22} & \bar{u} \bar{Q}_{13}+\bar{Q}_{23} \\
0 & \bar{u} \bar{Q}_{22} & \bar{u} \bar{Q}_{23}+\bar{Q}_{33}
\end{array}\right) .
$$

Again, we work column by column and compare entries, using the facts that the set $\left\{1, \bar{u}, \bar{u}^{2}\right\}$ is linearly independent over $k$ and that the $P_{i j}$ have entries in $k$. In this case, we find that $\bar{Q}_{i j}=0$ if $i \neq j$ and that $\bar{Q}_{11}=\bar{Q}_{22}=\bar{Q}_{33}=P_{11}$. Putting $\Delta:=P_{11}$, we have

$$
\bar{\theta}^{\prime}=\left(\begin{array}{ccc}
\Delta & 0 & 0 \\
0 & \Delta & 0 \\
0 & 0 & \Delta
\end{array}\right)
$$

Also as in the proof of Theorem 2.3, we can construct the commutative cube (2.3.7). (Note that $B$ is equivalent to $\left(\begin{array}{cc}H-\bar{u} I & 0 \\ 0 & \bar{u} I\end{array}\right)$, and is therefore invertible.) Exactly as in the proof of Theorem 2.3 , we write $\tilde{\theta}=\left(R_{i j}\right)$ and $\tilde{\theta}^{\prime}=\left(\tilde{Q}_{i j}\right)$ (again indexing the $\tilde{Q}_{i j}$ by the subscripts 2 and 3$)$. Then commutativity of the righthand trapezoid of (2.3.7) implies that $\tilde{Q}_{23} \sigma_{3}=\sigma_{2} Q_{23}$, from which we deduce that $\tilde{Q}_{23}=0$; that is, $\left(\tilde{Q}_{i j}\right)$ is block lower triangular. Commutativity of (2.3.7) also implies, for $i=2,3$, that $\tilde{Q}_{i i} \sigma_{i}=\sigma_{i} Q_{i i}$, and, exactly as in the proof of Theorem 2.3 , the diagonal form of the map $\sigma_{i}$ leads to the equality $\tilde{Q}_{i i}=\bar{Q}_{i i}=\Delta$. Now we invoke the equation $\tilde{\theta} B=B \tilde{\theta}^{\prime}$ (from the top trapezoid of (2.3.7)), getting

$$
\left(\begin{array}{ll}
R_{11} H+\bar{u}^{2} R_{12} & R_{11}+\bar{u} R_{12} \\
R_{21} H+\bar{u}^{2} R_{22} & R_{21}+\bar{u} R_{22}
\end{array}\right)=\left(\begin{array}{cc}
H \Delta+\tilde{Q}_{32} & \Delta \\
\bar{u}^{2} \Delta+\bar{u} \tilde{Q}_{32} & \bar{u} \Delta
\end{array}\right)
$$

Comparing the second columns of these matrices, we see that $\tilde{\theta}=\left(\begin{array}{cc}\Delta & 0 \\ 0 & \Delta\end{array}\right)$. Now from the first column, since $\bar{u}$ is a unit, we get that $\tilde{Q}_{32}=0$, and hence $\Delta H=H \Delta$. It follows that $\Delta$ is an upper-triangular matrix over $k$, with constant diagonal, and hence from (2.4.3) we see that $\bar{\theta}^{\prime}$ (which is the reduction modulo $\mathfrak{m}$ of the map $\theta^{\prime}$ ) is also an upper-triangular matrix with constant diagonal.

Finally, the last paragraph of $\S 2 \mathrm{~b}$ completes the proof of Theorem 2.4 , in the basic case.

There remain the two extreme possibilities not covered by the basic case. 
Inseparable case. (That is, $m=1$, and $F_{1}$ is of characteristic 2 and inseparable over $k$.) These hypotheses imply that $t=1$ and $\left[F_{1}: k\right] \geq 4$. By [Wi, Theorem 2.1] there is, for each positive integer $n$, an indecomposable finitely generated torsionfree $R$-module of rank $n$. Since it is not immediately clear how to modify the construction in [Wi] so as to obtain infinitely many non-isomorphic modules of each rank, we will again build mixed modules.

Using the fact that $\left[F_{1}: k\right] \geq 4$, we can select units $\bar{u}, \bar{v} \in D=\bar{R} / \mathfrak{m}$ such that the set $\{1, \bar{u}, \bar{v}, \bar{u} \bar{v}\}$ is linearly independent over $k$. Choose elements $u, v \in \bar{R}$ whose images in $D$ are $\bar{u}$ and $\bar{v}$, respectively, and set $\Omega:=R[u, v]$. As in the basic case, $\mathfrak{m}$ is the conductor of $R$ in $\Omega, \Omega / \mathfrak{m}$ is a finite-dimensional semisimple $k$-subalgebra of $D$, and $\mathfrak{m}=\mathrm{J}(\Omega)$.

The remainder of the proof in the inseparable case is almost identical to that of the basic case, except that we need to use a different matrix $A$ in the definition of the module $S$ in (2.4.2), and we need to use a different matrix $B$ in diagram (2.3.5) in order to define $\tau=B \sigma$ and $M_{n}=S / \operatorname{Im} \tau$. Specifically, let

$$
A=\left(\begin{array}{ccc}
I & 0 & 0 \\
\bar{u} I & I & 0 \\
\bar{v} I & 0 & I
\end{array}\right) \quad \text { and } \quad B=\left(\begin{array}{cc}
H & I \\
\bar{v} I & \bar{u} I
\end{array}\right)
$$

where $I$ is the $n \times n$ identity matrix, and $H$ is the nilpotent matrix in (2.3.4). The details of the proof in this case are left as an exercise for the interested reader.

Small residue field case. (That is, $\left[F_{1}: k\right]=1$, and $|k| \leq 3$.) Now the hypotheses imply that $m \geq 3$, so there are idempotents $\bar{e}_{1}=(1,0, \ldots, 0), \bar{e}_{2}=(0,1,0, \ldots, 0)$ and $\bar{e}_{3}=(0,0,1,0, \ldots, 0)$ in $D$. Choose elements $e_{1}, e_{2}, e_{3} \in \bar{R}$ whose images in $D$ are $\bar{e}_{1}, \bar{e}_{2}$, and $\bar{e}_{3}$, respectively, and set $\Omega=R\left[e_{1}, e_{2}, e_{3}\right]$. Then $\mathfrak{m}$ is the conductor of $R$ in $\Omega, \Omega / \mathfrak{m} \cong k \times k \times k$, and $\mathrm{J}(\Omega)=\mathfrak{m}$.

Just as in the basic case, fix elements $\lambda_{i} \in K_{i}^{\times} \cap R$ for each index $i$, set $w_{C}=$ $\sum_{i \notin C} \lambda_{i}$ for any subset $C \subseteq\{1, \ldots, t\}$, and choose subsets $C_{1}, \ldots, C_{n} \subseteq\{1, \ldots, t\}$ such that $i$ is in exactly $r_{i}$ sets $C_{j}$ for each $i$. Then the module $X_{1}:=\Omega / w_{C_{1}} \mathfrak{m}^{5} \oplus$ $\ldots \oplus \Omega / w_{C_{n}} \mathfrak{m}^{5}$ has torsion-free rank $\left(r_{1}, \ldots, r_{t}\right)$, and the natural map $\Omega^{(n)} \rightarrow$ $(\Omega / \mathfrak{m})^{(n)}$ provides an identification $X_{1} / \mathfrak{m} X_{1}=(\Omega / \mathfrak{m})^{(n)}$. Again, since $\Omega / \mathfrak{m}$ is a semisimple $k$-algebra, there is an $\Omega$-module $Y_{s}$ as in (2.4.1), for each positive integer $s$. We put $X_{2}:=\left(\Omega / Y_{4}\right)^{(n)}$ and $X_{3}:=\left(\Omega / Y_{2}\right)^{(n)}$; then the natural map $\Omega^{(n)} \rightarrow$ $(\Omega / \mathfrak{m})^{(n)}$ allows the identifications $X_{2} / \mathfrak{m} X_{2}=(\Omega / \mathfrak{m})^{(n)}$ and $X_{3} / \mathfrak{m} X_{3}=(\Omega / \mathfrak{m})^{(n)}$. Let $X=X_{1} \oplus X_{2} \oplus X_{3}$, and let $\nu: X \rightarrow X / \mathfrak{m} X=(\Omega / \mathfrak{m})^{(3 n)}$ be the natural map.

We define an $R$-module $S$ by means of the pullback square (2.4.2), where now the elements of $k^{(3 n)}$ are row vectors subjected to right multiplication by the matrix

$$
A=\bar{e}_{1} \cdot\left(\begin{array}{ccc}
I & 0 & 0 \\
0 & I & 0 \\
0 & 0 & I
\end{array}\right)+\bar{e}_{2} \cdot\left(\begin{array}{ccc}
0 & I & 0 \\
I & 0 & 0 \\
0 & 0 & I
\end{array}\right)+\bar{e}_{3} \cdot\left(\begin{array}{ccc}
0 & 0 & I \\
0 & I & 0 \\
I & 0 & 0
\end{array}\right),
$$

and $I$ again denotes the $n \times n$ identity matrix.

As in the basic case, $S$ is a separated $R$-module, and $\Omega \otimes_{R} S=\Omega S=X$. Moreover, we can fix injections $\xi: \Omega / \mathfrak{m} \rightarrow \Omega / Y_{4}$ and $\eta: \Omega / \mathfrak{m} \rightarrow \Omega / Y_{2}$ such that $\operatorname{Im} \xi=\mathfrak{m}^{3} / Y_{4}$ and $\operatorname{Im} \eta=\mathfrak{m} / Y_{2}$. Let $\sigma_{2}$ be the direct-sum map $\xi^{(n)}:(\Omega / \mathfrak{m})^{(n)} \rightarrow$ $\left(\Omega / Y_{4}\right)^{(n)}=X_{2}$ and $\sigma_{3}$ be the direct-sum map $\eta^{(n)}:(\Omega / \mathfrak{m})^{(n)} \rightarrow\left(\Omega / Y_{2}\right)^{(n)}=X_{3}$. Also as in the basic case, $\sigma_{2}$ and $\sigma_{3}$ are both injective, and $\operatorname{Im} \sigma_{2}=\left(\mathfrak{m}^{3} / Y_{4}\right)^{(n)}=$ 
$\mathfrak{m}^{3} X_{2}$ and $\operatorname{Im} \sigma_{3}=\left(\mathfrak{m} / Y_{2}\right)^{(n)}=\mathfrak{m} X_{3}$. So again $\operatorname{Im} \sigma_{2} \oplus \operatorname{Im} \sigma_{3} \subset \mathfrak{m} S$, and we get the commutative diagram (2.3.3) with $\sigma=\left(\begin{array}{ccc}0 & \sigma_{2} & 0 \\ 0 & 0 & \sigma_{3}\end{array}\right)$.

Let $B: k^{(2 n)} \rightarrow(\Omega / \mathfrak{m})^{(2 n)}$ be right multiplication by the matrix

$$
B=\bar{e}_{1} \cdot\left(\begin{array}{ll}
I & 0 \\
0 & I
\end{array}\right)+\bar{e}_{2} \cdot\left(\begin{array}{cc}
0 & I \\
I & 0
\end{array}\right)+\bar{e}_{3} \cdot\left(\begin{array}{cc}
I & H \\
0 & I
\end{array}\right)
$$

where $H$ is the nilpotent matrix in (2.3.4). Define $\tau$ to be the composition $B \sigma$ : $k^{(2 n)} \rightarrow S$ as in (2.3.5), and put $M_{n}=S / \operatorname{Im} \tau$. Again as in the basic case, $M_{n}$ has torsion-free rank $\left(r_{1}, \ldots, r_{t}\right)$, from the choice of $w_{C_{1}}, \ldots, w_{C_{n}}$; moreover, $M_{n} / \mathfrak{m} M_{n} \cong k^{(3 n)}$, yielding an infinite sequence of pairwise non-isomorphic $R$ modules, each with torsion-free rank $\left(r_{1}, \ldots, r_{t}\right)$.

The proof that $M_{n}$ is indecomposable proceeds much the same as in the proof of the preceding case, using commutative cubes analogous to (2.3.6) and (2.3.7). In this case, however, we make repeated use of the fact that the $\bar{e}_{i}$ are orthogonal idempotents with sum 1 , rather than the linear independence of the units $1, \bar{u}$, and $\bar{u}^{2}$. The first part of the argument, which uses the equation $\bar{\theta} A=A \bar{\theta}^{\prime}$, leads to an equation slightly different from (2.4.3), namely

$$
\bar{\theta}^{\prime}=\left(\begin{array}{ccc}
\Delta_{1} & 0 & 0 \\
0 & \Delta_{2} & 0 \\
0 & 0 & \Delta_{3}
\end{array}\right),
$$

where $\Delta_{1}, \Delta_{2}, \Delta_{3}$ are matrices over $\Omega / \mathfrak{m}$ satisfying the following equations:

$$
\begin{aligned}
& \bar{e}_{1} \Delta_{1}=\bar{e}_{2} \Delta_{2}=\bar{e}_{3} \Delta_{3}, \\
& \bar{e}_{1} \Delta_{2}=\bar{e}_{2} \Delta_{1}=\bar{e}_{3} \Delta_{2}, \\
& \bar{e}_{1} \Delta_{3}=\bar{e}_{2} \Delta_{3}=\bar{e}_{3} \Delta_{1} .
\end{aligned}
$$

The second part of the argument, which uses the equation $\tilde{\theta} B=B \tilde{\theta}^{\prime}$, yields the equations

$$
\begin{gathered}
\bar{e}_{1} \Delta_{2}=\bar{e}_{2} \Delta_{3}=\bar{e}_{3} \Delta_{2} \\
\bar{e}_{3} \Delta_{2} H=\bar{e}_{3} H \Delta_{3} .
\end{gathered}
$$

Combining (2.4.4) and (2.4.5), we easily deduce that $\Delta_{1}=\Delta_{2}=\Delta_{3}=: \Delta$ is in fact a matrix over $k$, and that $\Delta H=H \Delta$.

We note two more important facts concerning our choice of the matrix $B$. First, from the form of the matrix $B$, one checks easily that no non-zero $\Omega$-submodule of $X$ is contained in $\operatorname{Im} \tau=\operatorname{Im}(B \sigma)$. Since $\operatorname{Im} \tau \subseteq \mathfrak{m} S, S$ is a separated cover of $M_{n}$. Second, one also checks easily that $B$ is invertible, so that $\operatorname{Im}(B \sigma)$ generates $\operatorname{Im} \sigma$ as an $\Omega$-submodule of $X$. Therefore the $\Omega$-homomorphism $\theta^{\prime}$ lifts to the $\Omega$ homomorphism $\tilde{\theta}^{\prime}$ in (2.3.7).

The remaining details of the proof in this case are also left as an exercise for the interested reader.

\section{§3. Rings of Higher Dimension AND NON-COHEN-MaCAUlay RINGS}

If $R$ is a one-dimensional local ring that is not Cohen-Macaulay, then $R$ is equal to its own total quotient ring $K$. Therefore there is no hope of building indecomposable generically free (that is, projective) $R$-modules of large rank. Thus, in the nonCohen-Macaulay case, we use another measure of the size of a module $M$, namely, the multiplicity $\mathrm{e}_{R}(M)$. Recall [M, p. 107] that, for a finitely generated module $M$ 
over a $d$-dimensional local ring $(R, \mathfrak{m}, k)$, the multiplicity is defined by $\mathrm{e}_{R}(M)=$ $\mathrm{e}_{R}(\mathfrak{m}, M)=\lim _{n \rightarrow \infty} \frac{d !}{n^{d}} \ell_{R}\left(M / \mathfrak{m}^{n} M\right)$. (Here $\ell_{R}$ denotes length as an $R$-module.) From [M, Theorem 14.7], we have the following:

Remark 3.1. If $M$ has constant rank $r, \mathrm{e}_{R}(M)=r \cdot \mathrm{e}_{R}(R)$.

Thus the existence of indecomposables of large constant rank implies that there are indecomposables with large multiplicity; for domains the converse is true.

There are two obstacles to determining which one-dimensional local rings have a bound on the multiplicities of indecomposable finitely generated modules. One is the irksome case of an imperfect residue field of characteristic 2, which disrupts the tame-wild dichotomy in [KL3]. Another is that our construction of large indecomposable modules seems not to extend to one-dimensional non-CohenMacaulay rings. Note that there exist one-dimensional local rings, e.g., $R=$ $\mathbb{C}[[X, Y]] /\left(X^{2}, X Y\right)$, that are not Dedekind-like but that can be expressed in the form $D / I$ for a suitable Dedekind-like ring $D$ and non-zero ideal $I$. Such rings cannot be Cohen-Macaulay. (To see this, note that $D$ is not a domain and thus has exactly two minimal prime ideals $P$ and $Q$, with $P \cap Q=0$. Since $\operatorname{dim}(D / I)=1$, we may assume that $(0) \subsetneq I \subsetneq P$ and $I \nsubseteq Q$. Then $(P / I)_{P}=(0)$ and $(P / I)_{Q}=(0)$. Therefore $P / I$ is a "non-zero finite-length submodule of $D / I$, and $\operatorname{depth}(D / I)=0$.) Since $D$ is not a domain, the separability condition from the second paragraph of the introduction is vacuously satisfied. The results of [KL2] then show that every indecomposable finitely generated $D$-module has torsion-free rank $(1,0),(0,1)$ or $(1,1)$, and it follows that the multiplicity of every indecomposable finitely generated $D / I$-module is at most 2 .

On the other hand, there are one-dimensional local non-Cohen-Macaulay rings, for example, $R=\mathbb{C}[[X, Y]] /\left(X^{2}, X Y^{2}\right)$, that are not homomorphic images of Dedekind-like rings. (This ring maps onto the Drozd ring $\mathbb{C}[[X, Y]] /\left(X^{2}, X Y^{2}, Y^{3}\right)$, and therefore by [KL1, Theorem 3.1] is not a homomorphic image of a Dedekind-like ring.) Using completely different methods, the authors of the present paper have recently shown that every one-dimensional non-Cohen-Macaulay ring that is not a homomorphic image of a Dedekind-like ring has indecomposable finitely generated modules of arbitrarily large multiplicity. This theorem implies the following result, which almost characterizes the local rings having a bound on the multiplicities of finitely generated modules. We refer the reader to our forthcoming paper [HKKW] for the details.

Theorem 3.2. Suppose $(R, \mathfrak{m}, k)$ is a local ring such that there is a bound on the multiplicities of the indecomposable finitely generated $R$-modules.

(1) Either $\operatorname{dim}(R)=1$, or else $R$ is an Artinian principal ideal ring (in which case every indecomposable finitely generated module is cyclic and hence has multiplicity at most $\left.\mathrm{e}_{R}(R)=\ell_{R}(R)\right)$.

(2) Assume $R$ is one-dimensional. Then $R$ is a homomorphic image of a Dedekind-like ring. If, further, $k$ is perfect or of characteristic different from 2 , then every indecomposable $R$-finitely generated module has multiplicity bounded by 4 . 


\section{REFERENCES}

[B] H. Bass, On the ubiquity of Gorenstein rings, Math. Z. 82 (1963), 8-28. MR0153708 $(27: 3669)$

[CWW] N. Çimen, R. Wiegand and S. Wiegand, One-dimensional rings of finite representation type, Abelian groups and modules (Padova, 1994), Kluwer Acad. Publ., Dordrecht, 1995, pp. 95-121. MR1378192 (97a:13014)

[DR] Ju. A. Drozd and A. V. Roüter, Commutative rings with a finite number of indecomposable integral representations (Russian), Izv. Akad. Nauk. SSSR, Ser. Mat. 31 (1967), 783-798. MR0220716 (36:3768)

[HKKW] W. Hassler, R. Karr, L. Klingler and R. Wiegand, Large indecomposable modules over local rings, J. Algebra 303 (2006), 202-215. MR2253659

[HaW] W. Hassler and R. Wiegand, Direct sum cancellation for modules over one-dimensional rings, J. Algebra 283 (2005), 93-124. MR2102074 (2006b:13030)

[KL1] L. Klingler and L. S. Levy, Representation type of commutative Noetherian rings I: Local Wildness, Pacific J. Math. 200 (2001), 345-386. MR1868696 (2002i:13008a)

[KL2] L. Klingler and L. S. Levy, Representation type of commutative Noetherian rings II: Local Tameness, Pacific J. Math. 200 (2001), 387-483. MR1868697 (2002i:13008b)

[KL3] L. Klingler and L. S. Levy, Representation type of commutative Noetherian rings III: Global Wildness and Tameness, Mem. Amer. Math. Soc. 176 (2005), no. 832. MR2147090 (2006g:13037)

[LevW] L. S. Levy and R. Wiegand, Dedekind-like behavior of rings with 2-generated ideals, J. Pure Appl. Algebra 37 (1985), 41-58. MR794792 (86k:13012)

[LW1] G. Leuschke and R. Wiegand, Hypersurfaces of bounded Cohen-Macaulay type, J. Pure Appl. Algebra 201 (2005), 204-217. MR2158755 (2006c:13014)

[LW2] Local rings of bounded Cohen-Macaulay type, Algebr. Represent. Theory 8 (2005), 225-238. MR2162283 (2006c:13013)

[M] H. Matsumura, Commutative Ring Theory, Cambridge Stud. Adv. Math., vol. 8, Cambridge University Press, Cambridge, 1986. MR879273 (88h:13001)

[Wa] R. Warfield, Decomposability of finitely presented modules, Proc. Amer. Math. Soc. 25 (1970), 167-172. MR0254030 (40:7243)

[Wi] R. Wiegand, Noetherian rings of bounded representation type, Commutative Algebra, Proceedings of a Microprogram (June 15 - July 2, 1987), Springer-Verlag, New York, 1989, pp. 497-516. MR1015536 (90i:13010)

Institut für Mathematik und wissenschaftliches ReChnen, KarL-Franzens-Universiтёт Graz, Heinrichstrasse 36/IV, A-8010 Graz, Austria

Honors College, Florida Atlantic University, Jupiter, Florida 33458

Department of Mathematical Sciences, Florida Atlantic University, Boca Raton, FLORIDA 33431-6498

Department of Mathematics, University of Nebraska-Lincoln, Lincoln, Nebraska 68588-0130 